\title{
A Mathematical Model of the Formation of Lanes in Crowds of Pedestrians Moving in Opposite Directions
}

\author{
Guillermo H. Goldsztein \\ School of Mathematics, Georgia Institute of Technology, 686 Cherry Street, Atlanta, GA 30332-0160, USA \\ Correspondence should be addressed to Guillermo H. Goldsztein; ggold@math.gatech.edu
}

Received 5 May 2015; Accepted 9 June 2015

Academic Editor: Tetsuji Tokihiro

Copyright ( 2015 Guillermo H. Goldsztein. This is an open access article distributed under the Creative Commons Attribution License, which permits unrestricted use, distribution, and reproduction in any medium, provided the original work is properly cited.

In crowded environments, pedestrians moving in opposite directions segregate into lanes of individuals moving in the same direction. It is believed that this formation of lanes that facilitates the flow results from the individuals acting on their behalf, responding to local stimuli, without the intention of benefiting the crowd as a whole. We give evidence that this is true by developing and analyzing a simple mathematical model. Our results suggest that the simple behavior of moving out of the way to avoid imminent collisions leads to the formation of lanes of individuals moving in the same direction.

\section{Introduction}

Self-organization refers to the pattern formation or emergence of order in systems composed of several units or individuals, where these units or individuals respond only to local stimuli, independently of the global state of the system.

Primary research goals in the field of self-organization are to understand the local interactions among individuals and with the environment and how these local interactions lead to the global self-organization. A major objective is to discover common features of different self-organizing systems to establish general principles that apply to classes of systems. Some books and review articles on self-organization in biological systems include [1-3].

Self-organization in human crowds is commonly observed in real life scenarios and is well documented in the literature. Examples include the occurrence of alternating flows through bottlenecks, as two groups of a large number of individuals cross the bottleneck in opposite directions [4], the formation of trails [5], and the different pattern formations when the crowd is a collection of several small groups (friends) [6]. These coordinated behaviors are not planned by the crowd as a whole or by any member or group of members within the crowd. Instead, they emerge as a consequence of the individuals acting in response to local stimuli and motivated by their own goals.
Examples of why the understanding of the dynamics of crowds is of interest include the following: (1) It is a source of interesting examples of self-organization. (2) It can provide guidelines to increase the safety in very transited areas like some bridges [7] or in events attended by massive crowds, such as events in stadiums. (3) It can provide guidelines in the design of buildings, such as movie theaters and shopping malls, where optimal crowd flow is desired because of economical and safety reasons. Accordingly, the study of dynamics of crowds, both theoretically (early works include $[8,9]$; see also [10] for a review) and experimentally [11-13], is a very active area of research.

The existing mathematical models of crowd dynamics can be categorized according to the length scale modeled as microscopic, mesoscopic, and macroscopic.

Microscopic models solve for the position of each individual. This class includes cellular automata models [14-16], lattice gas automata models $[17,18]$, algorithms [19], and large systems of odes, where the mass times acceleration of each individual is set equal to the sum of generalized or social forces the individual feels [20-24]. These social forces are not real forces between individuals but are introduced to model the responses of the individuals to stimuli they feel, which include the environment and the presence of the other individuals. In most models, the social forces are of four types: (1) repulsive force acting in a very short range, to avoid collisions with 
other individuals or walls or obstacles, (2) attractive force that acts in a longer range and attempts to model the desire of individuals to move in groups, (3) a force that would result in the desired speed and direction of motion of the individual in the absence of the other forces, and (4) a random force to account for differences in individuals. In some models, the speed of each individual remains constant, and only its direction changes in response to the social forces [25]. Some of these models are known as self-propelled particles models [26] and others as individual based models [25, 27].

Mesoscopic, kinetic, or Boltzmann type models are integropartial differential equations that describe the evolution of probability densities of the position and velocities of the pedestrians $[28,29]$. Macroscopic or continuum models are partial differential equations (conservation equations), where the dependent variables are the density and local average velocity of individuals [30-34]. Some works connect microscopic to macroscopic models [35-37]. Also, network models have been introduced [38] and optimal control theory has been used [39].

Consider a crowded long corridor or street. Assume some of the pedestrians move in one direction and the others in the opposite direction. A self-organizing phenomenon familiar to all of us is that the individuals segregate into lanes, where each lane contains only individuals moving in one direction [40-42]. This formation of lanes facilitates the flow in both directions [43]. In this paper, we introduce and analyze a new model motivated by this phenomenon. This new model is very different to the ones that can be found in the literature. The results we obtain suggest that the simple behavior of moving out of the way to avoid collisions leads to the selforganization of the system.

In Section 2, we introduce our model, in which we assume that individuals move around a two-lane circular track with the same angular speed, half of them in one direction and the other half in the opposite direction. Each time two individuals moving in the opposite direction and in the same lane meet; one of them, randomly chosen with each having the same probability of $1 / 2$ of being chosen, moves to the other lane so they both can continue their motion around the track. In Section 3, we prove that, with probability 1 , the system will self-organize; that is, all the individuals moving in the counterclockwise direction end up in the same lane, and all other individuals, moving in the clockwise direction, end up in the other lane. More details on the dynamics and final state of our system are given in the subsequent sections. In Section 4 , we provide the probability that, after the system is self-organized, the pedestrians in lane 1 move in the clockwise direction. In Section 5, we find the expected number of collisions required for the system to self-organize, and in Section 6 we compute numerically the expected time to selforganization. We finish the paper with a small discussion in Section 7.

\section{The Mathematical Model}

Assume that $N$ individuals or pedestrians move around a two-lane circular track, half of them in one direction and the other half in the opposite direction. Each time two individuals moving in opposite directions and in the same lane meet; one of them, randomly chosen, with each having the same probability of $1 / 2$ of being chosen, moves to the other lane so they both can continue their motion around the track. More precisely, our model is defined as follows.

Rules. The following set of rules define the dynamics of our model.

(1) $N$ individuals move around a circular track with two lanes. The inner lane is referred to as lane 1 and the outer lane as lane 2. Each individual is labeled with an integer between 1 and $N$. We assume $N$ is even.

(2) $N / 2$ individuals move in the counterclockwise direction and the other $N / 2$ in the clockwise direction.

(3) All individuals move with the same constant angular speed $\omega$.

(4) The position of the individual $i$ at time $t$ is described by an angle $\theta_{i}(t)$ and by $\ell_{i}(t)$, the number of the lane the individual $i$ is in at time $t$. Note that the angle $\theta_{i}(t)$ is not uniquely determined by the position of the individual $i$. Changing $\theta_{i}(t)$ by adding to it an integer multiple of $2 \pi$ does not change the position of the individual $i$.

(5) We take all the angles to satisfy $0 \leq \theta_{i}(t)<2 \pi$ for all $t$. The angle $\theta_{i}(t)$ is assumed to increase counterclockwise. Thus, if the individual $i$ moves in the counterclockwise direction, we have $\theta_{i}(t)=\theta_{i}(0)+$ $\omega t-2 \pi M_{i}(t)$, where $M_{i}(t)$ is only the integer (that depends on $t)$ that makes $\theta_{i}(t)$ satisfy the constraint $0 \leq \theta_{i}(t)<2 \pi$. Analogously, if the individual $i$ moves in the clockwise direction, then $\theta_{i}(t)=\theta_{i}(0)-\omega t+$ $2 \pi M_{i}(t)$ for an integer value function $M_{i}(t)$. With this rule, $\theta_{i}(t)$ is uniquely determined.

(6) We assume that initially all the angles are different; that is, $\theta_{i}(0) \neq \theta_{j}(0)$ for all $i \neq j$.

(7) Two individuals, $i$ and $j$, collide at time $t$ if they reach the same location at that time; that is, $\theta_{i}(t)=\theta_{j}(t)$ and $\ell_{i}(t)=\ell_{j}(t)$ (they have the same angle and are in the same lane).

(8) If two individuals, $i$ and $j$, collide at time $t$, exactly one of them changes lanes at that time. The probability that the individual $i$ changes lanes is $1 / 2$ and, thus, $1 / 2$ is also the probability that $j$ changes lanes. The lane change is instantaneous; that is, it does not slow down the angular speed of the individual changing lanes.

(9) An individual can only change lanes when it collides with another individual.

Observation 1. Note that the assumptions that all the initial angles are different and that the angular speed of all individuals is the same imply that two individuals moving in the same direction will never have the same angle. Thus, if two individuals have the same angle at some time $t$, they must be moving in opposite directions. These facts imply that when two individuals collide, there is no other individual next to them in the other lane, and, thus, our rule that one of 


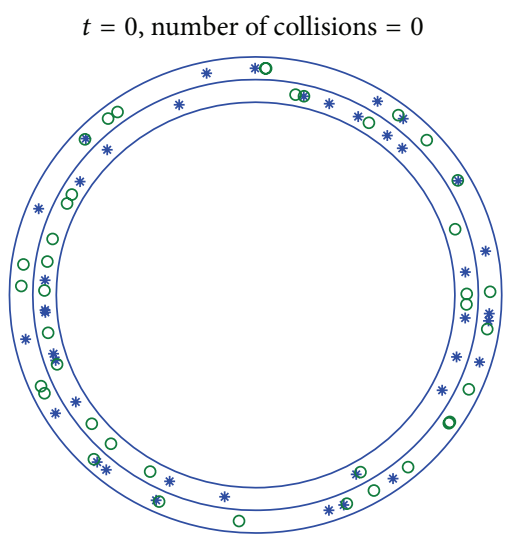

(a)

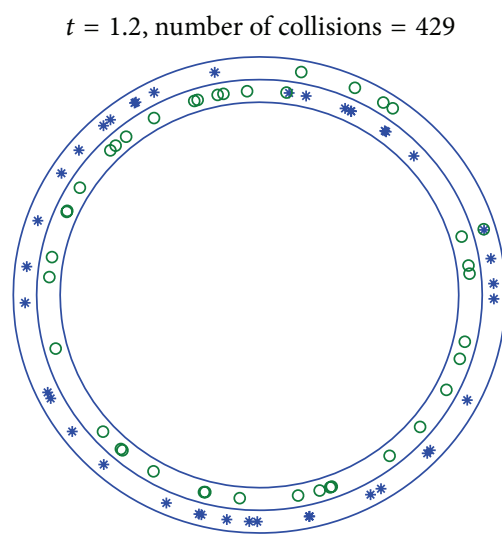

(b)

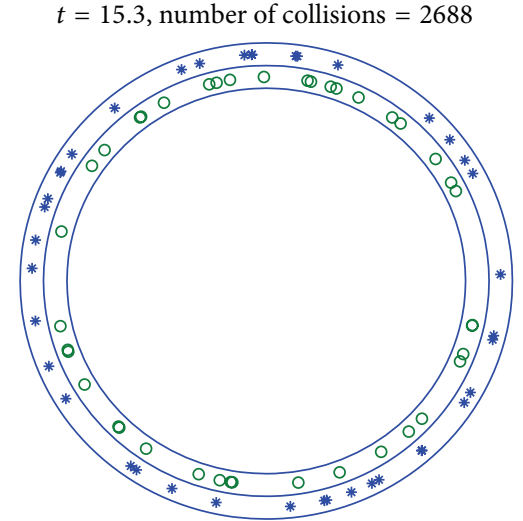

(c)

FIGURE 1: Positions of the pedestrians at different times. The circles are the pedestrians moving clockwise. The stars are the pedestrians moving counterclockwise.

them changes lanes can be followed with no problems and no exceptions.

Our model is very simple and is based on local responses only. The selection of the individual that changes lanes is independent of the location of all the other individuals. In other words, the individuals do not make smart decisions attempting to minimize the number of lane changes they will have to make.

Figure 1 shows the results of a numerical simulation with $N=80$ individuals. The individuals move at one revolution per unit time. Figure 1 shows the positions of the individuals at three different times: $t=0, t=1.2$, and $t=15.3$. The number of collisions that occurred by those times is also indicated in Figure 1. The initial angle of the individuals, $\theta_{i}(0)$, was randomly chosen with uniform probability distribution around the track. The lane where each pedestrian started, $\ell_{i}(0)$, was also randomly selected, with each lane having $1 / 2$ as the probability of being chosen.
Note that at $t=15.3$, after 2688 collisions, all the pedestrians moving in the counterclockwise direction are in the outer lane, and all those moving in the clockwise direction are in the inner lane. No more collisions occur after this time. We say that the system has self-organized. We have run several simulations with different randomly generated initial conditions (not shown here), and in all those simulations the system self-organized.

Our numerical simulations suggest that the system always self-organizes. Next, we prove that this is the case with probability 1 .

\section{The System Self-Organizes with Probability 1}

We denote by $t_{i}$ the time when the $i$ th collision occurs. For convenience, we also define $t_{0}=0$. We denote by $t_{i}^{+}$the time right after the $i$ th collision, that is, right once one of the individuals that collided changed lanes, and set $t_{0}^{+}=0$. We define

$$
\begin{aligned}
A_{i}= & \text { number of individuals in lane } 1 \text { moving counterclockwise at time } t_{i}^{+} \\
& \text {- number of individuals in lane } 1 \text { moving clockwise in the same time. }
\end{aligned}
$$

Observation 2. The following observations about the sequence $A_{i}$ will be used in our analysis.

(1) Since there are a total of $N / 2$ individuals moving counterclockwise and $N / 2$ moving clockwise, we have that $\left|A_{i}\right| \leq N / 2$ for all $i$.

(2) $A_{i}=N / 2$ implies that, at time $t_{i}^{+}$, all individuals moving in the counterclockwise direction are in lane 1 and all those moving clockwise are in lane 2 . Analogously, $A_{i}=-N / 2$ implies that, at time $t_{i}^{+}$, all those moving clockwise are in lane 1 and all those moving counterclockwise are in lane 2. Thus, if $\left|A_{i}\right|=N / 2$, there will not be any more collisions at later times, that is, for $t>t_{i}$. The system has selforganized.

(3) If $\left|A_{i}\right|<N / 2$, there will be at least one more collision at some time larger than $t_{i}$.

Given the above observations, proving that the system self-organizes is equivalent to proving that $\left|A_{i}\right|=N / 2$ for some $i$.

In what follows, we use the standard notations of $P(B)$ to denote the probability that the event $B$ occurs and $P(C \mid D)$ 
to denote the conditional probability that the event $C$ occurs given that the event $D$ has occurred.

Because all the individuals move with the same angular speed, any collision is between two individuals moving in opposite directions. Assume that the $i$ th collision occurs in lane 1. If the individual changing lanes to lane 2 is the one moving in the counterclockwise direction, we have $A_{i}=$ $A_{i-1}-1$; otherwise, $A_{i}=A_{i-1}+1$. Since each of the two individuals colliding has a probability of $1 / 2$ to change lanes, we have that

$$
\begin{aligned}
& P\left(A_{i}=A_{i-1}-1 \mid i \text { th collision was in lane } 1\right)=\frac{1}{2}, \\
& P\left(A_{i}=A_{i-1}+1 \mid i \text { th collision was in lane } 1\right)=\frac{1}{2} .
\end{aligned}
$$

Similar arguments show that

$$
\begin{aligned}
& P\left(A_{i}=A_{i-1}-1 \mid i \text { th collision was in lane } 2\right)=\frac{1}{2}, \\
& P\left(A_{i}=A_{i-1}+1 \mid i \text { th collision was in lane } 2\right)=\frac{1}{2} .
\end{aligned}
$$

As a consequence, we have

$$
P\left(A_{i}=A_{i-1}-1\right)=P\left(A_{i}=A_{i-1}+1\right)=\frac{1}{2} .
$$

Thus, we can identify $A_{i}$ with a random walk in $\mathbb{Z}$, the set of integers. The walker starts at $A_{0}$. When the $i$ th collision occurs, the walker moves to the right if $A_{i}=A_{i-1}+1$ or moves to the left otherwise. Thus, each time a collision occurs, the walker moves to the right with probability $1 / 2$ or to the left also with probability $1 / 2$, and the position of the walker right after the $i$ th collision is $A_{i}$.

We can now use the theory of random walks [44] to obtain conclusions about our model. Let

$$
K=\min \left\{i:\left|A_{i}\right|=\frac{N}{2}\right\} .
$$

Note that $K$ is the total number of collisions. After the $K$ th collision, the system is self-organized and no more collisions occur. It is well known that [44, page 14$]$

$$
P(K<\infty)=1 \text {. }
$$

Since the time between consecutive collisions is bounded (by half the time an individual takes to go around the track once), the last equation proves that the system self-organizes with probability one in finite time.

Observation 3. Note that we have proved that the system selforganizes with probability 1 regardless of the initial condition, that is, the initial angles $\theta_{i}(0)$ and the initial lane position $\ell_{i}(0)$ of the individuals.

In Figure 2, we show the evolution of $A_{i}$, corresponding to the same simulation as in Figure 1, as a function of $i$ and also as a function of time $t$. In this case, $A_{2688}=-N / 2=-40$. After this last collision, all the pedestrians in lane 1 (the inner lane) are those moving clockwise, and all the pedestrians in lane 2 are those moving counterclockwise.

\section{Direction of the Pedestrians in Each Lane after Self-Organization}

Known facts about random walks provide us with more information about the dynamics of our system. In particular, we have that [44, page 15]

$$
P\left(A_{K}=\frac{N}{2}\right)=\frac{\left(A_{0}+N / 2\right)}{N} ;
$$

that is, the probability that after the last collision all individuals in lane 1 move in the counterclockwise direction is the above a linear function of $A_{0}$. Note that this is an increasing function of $A_{0}$, which is expected since $A_{0}$ is the difference between the initial number of individuals in lane 1 moving counterclockwise and the initial number of individuals in lane 1 moving clockwise.

\section{Number of Collisions Required for Self-Organization}

Given some initial conditions and, thus, the value of $A_{0}$, the expected number of collisions needed to reach selforganization, $E\left[K \mid A_{0}\right.$ ], follows also from known facts about random walks [44, page 30$]$ :

$$
E\left[K \mid A_{0}\right]=\left(\frac{N}{2}-A_{0}\right)\left(A_{0}+\frac{N}{2}\right)
$$

(see (5) for a reminder of the definition of $K$ ).

Note that the value of (8) depends on the initial conditions through $A_{0}$. However, if we assume that each lane has a probability of $1 / 2$ to be the initial lane of each individual, as we did in all our numerical calculations, it can be shown that the law of large numbers implies that the expected number of collisions needed to reach self-organization, $E[K]$, is

$$
E[K]=\frac{N^{2}}{4}-O(N)
$$

where $O(N)$ means a quantity of order $N$, that is, bounded by some constant times $N$.

\section{Time Required for Self-Organization}

A final question we seek to answer is, What is the expected time required for the system to self-organize?

We have performed numerical simulations and obtained the results displayed in Figure 3. Each data point in that figure is the average value of 10,000 realizations. The initial positions of the individuals were randomly chosen with uniform probability distribution around the track. The lane where each pedestrian started was also randomly selected, with each lane having $1 / 2$ as the probability of being chosen. A least square fit of our data into a straight line gave us the straight line $E\left[T_{\text {org }}\right]=0.1013 N+0.1193$, where $N$ is the number of pedestrians and $E\left[T_{\text {org }}\right]$ is the expected time to self-organization. Our simulations suggest that $E\left[T_{\text {org }}\right]$ is asymptotically a linear function of $N$, the number of pedestrians, in the parameter regime of large $N$ (see Figure 3). 


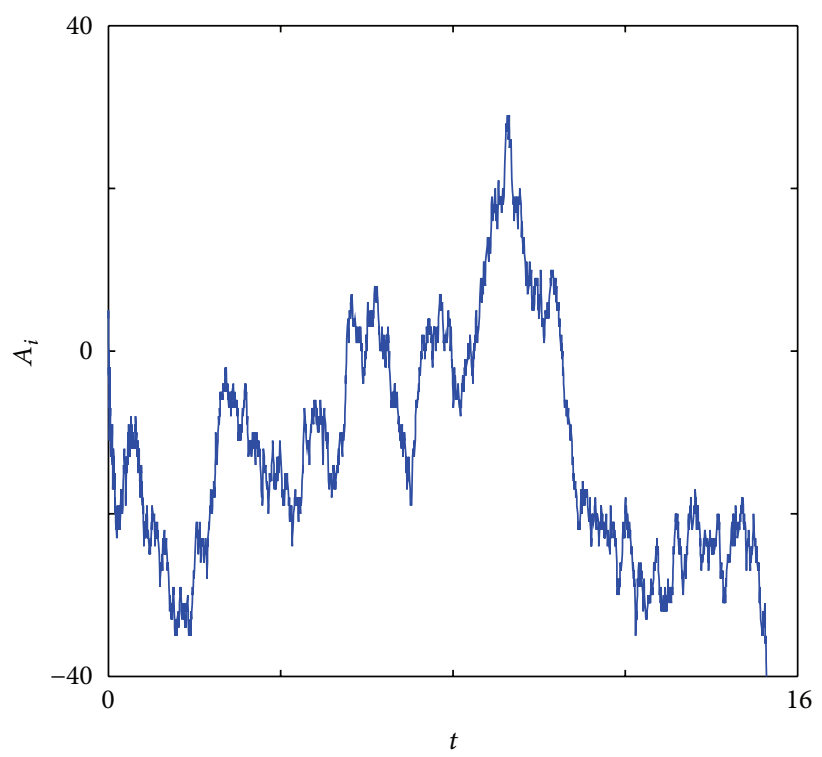

(a)

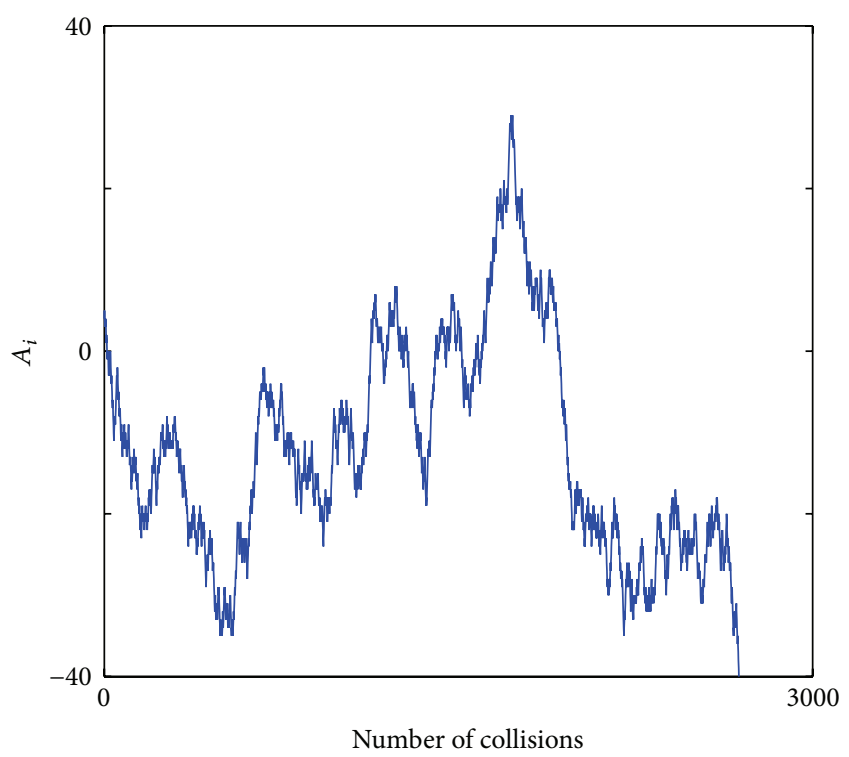

(b)

FIGURE 2: Evolution of the number of pedestrians moving counterclockwise minus the number of pedestrians moving clockwise in lane 1, the inner lane. In (a), we use the time $t$, but, in (b), we use the number of collisions as the independent variable.

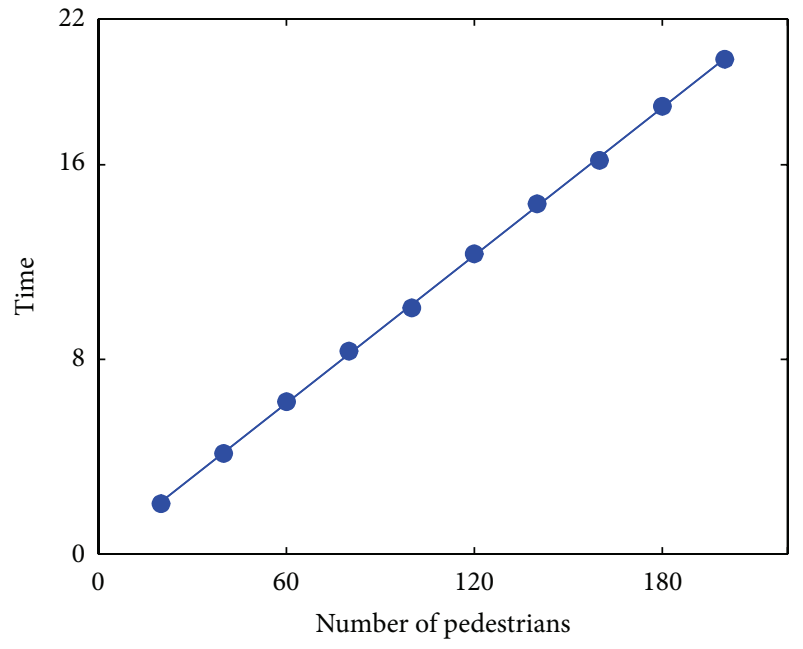

FIGURE 3: Expected time required for self-organization as a function of the number of pedestrians. The circles are the results of our simulations and the solid line is the straight line obtained with least squares fit.

\section{Summary}

We have introduced a very simple model to study the formation of lanes in crowds of individuals moving in opposite directions. The intent of our model is to isolate the effect of pedestrians avoiding collisions by simply moving out of the way only when the collision is imminent. Our model has proved simple enough to be amenable to analytical analysis. In fact, we were able to borrow many results from the theory of random walks in one dimension. Our results suggest that, in fact, this single behavior of moving out of the way only to avoid imminent collisions leads to the formation of lanes.
The model studied in this paper belongs to the class of microscopic models. However, it is truly different in nature from the existing models that can be found in the literature. This model is the simplest model we could think of that isolates the effect of pedestrians avoiding collisions in a very simple way. This model that could be considered a toy model has the usual advantage of simple models: (1) The results obtained are clear, concrete, and easy to interpret. (2) Its simplicity makes it amenable to analytical analysis.

There is a tradeoff, and with simplicity we sometimes lose generality. Most models in the literature do not lend themselves to the simple analytical analysis presented in this paper. Instead, they have to be solved numerically. However, they are more general in the sense that different phenomena can be studied by simply changing boundary or initial conditions. We believe that both strategies, the study of simple toy models, as in this paper, and the study of comprehensive more complex models, as most of the studies found in the literature, are very valuable and complement each other.

We believe that this paper is a step forward towards the understanding of self-organization in biological systems, and we hope the modeling style of this paper will be adopted by other researchers to study this and other self-organization phenomena in biological systems.

\section{Conflict of Interests}

The author declares that there is no conflict of interests regarding the publication of this paper.

\section{References}

[1] S. Camazine, Self-Organization in Biological Systems, Princeton University Press, Princeton, NJ, USA, 2003. 
[2] D. J. T. Sumpter, Collective Animal Behavior, Princeton University Press, 2010.

[3] T. Vicsek and A. Zafeiris, "Collective motion," Physics Reports, vol. 517, no. 3-4, pp. 71-140, 2012.

[4] T. Kretz, M. Wölki, and M. Schreckenberg, "Characterizing correlations of flow oscillations at bottlenecks," Journal of Statistical Mechanics: Theory and Experiment, vol. 2006, no. 2, article P02005, 2006.

[5] D. Helbing, J. Keltsch, and P. Molnár, "Modelling the evolution of human trail systems," Nature, vol. 388, no. 6637, pp. 47-50, 1997.

[6] M. Moussaïd, N. Perozo, S. Garnier, D. Helbing, and G. Theraulaz, "The walking behaviour of pedestrian social groups and its impact on crowd dynamics," PLOS ONE, vol. 5, no. 4, Article ID e10047, 2010.

[7] L. Bruno and F. Venuti, "Crowd-structure interaction in footbridges: modelling, application to a real case-study and sensitivity analyses," Journal of Sound and Vibration, vol. 323, no. 1-2, pp. 475-493, 2009.

[8] L. F. Henderson, “The statistics of crowd fluids," Nature, vol. 229, no. 5284, pp. 381-383, 1971.

[9] L. F. Henderson, "On the fluid mechanics of human crowd motion," Transportation Research, vol. 8, no. 6, pp. 509-515, 1974.

[10] D. Helbing, "Traffic and related self-driven many-particle systems," Reviews of Modern Physics, vol. 73, no. 4, pp. 1067-1141, 2001.

[11] M. Moussaïd, E. G. Guillot, M. Moreau et al., "Traffic instabilities in self-organized pedestrian crowds," PLoS Computational Biology, vol. 8, no. 3, Article ID e1002442, 2012.

[12] S. Hoogendoorn and W. Daamen, "Self-organization in pedestrian flow," in Traffic and Granular Flow '03, pp. 373-382, Springer, Berlin, Germany, 2005.

[13] M. Moussaïd, D. Helbing, S. Garnier, A. Johansson, M. Combe, and G. Theraulaz, "Experimental study of the behavioural mechanisms underlying self-organization in human crowds," Proceedings of the Royal Society B: Biological Sciences, vol. 276, no. 1668, pp. 2755-2762, 2009.

[14] J. Ma, W.-G. Song, J. Zhang, S.-M. Lo, and G.-X. Liao, "kNearest-Neighbor interaction induced self-organized pedestrian counter flow," Physica A: Statistical Mechanics and its Applications, vol. 389, no. 10, pp. 2101-2117, 2010.

[15] F. Weifeng, Y. Lizhong, and F. Weicheng, "Simulation of bi-direction pedestrian movement using a cellular automata model," Physica A: Statistical Mechanics and its Applications, vol. 321, no. 3-4, pp. 633-640, 2003.

[16] V. J. Blue and J. L. Adler, "Cellular automata microsimulation for modeling bi-directional pedestrian walkways," Transportation Research Part B: Methodological, vol. 35, no. 3, pp. 293-312, 2001.

[17] M. Muramatsu, T. Irie, and T. Nagatani, "Jamming transition in pedestrian counter flow," Physica A: Statistical Mechanics and its Applications, vol. 267, no. 3, pp. 487-498, 1999.

[18] H. Kuang, X.-L. Li, Y.-F. Wei, T. Song, and S.-Q. Dai, "Effect of following strength on pedestrian counter flow," Chinese Physics $B$, vol. 19, no. 7, Article ID 070517, 2010.

[19] P. G. Gipps and B. Marksjö, "A micro-simulation model for pedestrian flows," Mathematics and Computers in Simulation, vol. 27, no. 2-3, pp. 95-105, 1985.

[20] D. Helbing and P. Molnár, "Social force model for pedestrian dynamics," Physical Review E, vol. 51, no. 5, pp. 4282-4286, 1995.
[21] D. Helbing, "A mathematical model for the behavior of pedestrians," Behavioral Science, vol. 36, no. 4, pp. 298-310, 1991.

[22] L. Jian, Y. Lizhong, and Z. Daoliang, "Simulation of bidirection pedestrian movement in corridor," Physica A: Statistical Mechanics and its Applications, vol. 354, no. 1-4, pp. 619-628, 2005.

[23] A. Treuille, S. Cooper, and Z. Popović, "Continuum crowds," ACM Transactions on Graphics, vol. 25, no. 3, pp. 1160-1168, 2006.

[24] D. Helbing, L. Buzna, A. Johansson, and T. Werner, "Selforganized pedestrian crowd dynamics: experiments, simulations, and design solutions," Transportation Science, vol. 39, no. 1, pp. 1-24, 2005.

[25] P. Degond and J. Hua, "Self-organized hydrodynamics with congestion and path formation in crowds," Journal of Computational Physics, vol. 237, pp. 299-319, 2013.

[26] T. Vicsek, A. Czirók, I. J. Farkas, and D. Helbing, "Application of statistical mechanics to collective motion in biology," Physica A: Statistical Mechanics and its Applications, vol. 274, no. 1, pp. 182-189, 1999.

[27] P. Degond, C. Appert-Rolland, M. Moussaïd, J. Pettré, and G. Theraulaz, "A hierarchy of heuristic-based models of crowd dynamics," Journal of Statistical Physics, vol. 152, no. 6, pp. 10331068, 2013.

[28] D. Helbing, "A fluid dynamic model for the movement of pedestrians," http://arxiv.org/abs/cond-mat/9805213.

[29] N. Bellomo, C. Bianca, and M. Delitala, "Complexity analysis and mathematical tools towards the modelling of living systems," Physics of Life Reviews, vol. 6, no. 3, pp. 144-175, 2009.

[30] C. Appert-Rolland, P. Degond, and S. Motsch, "Two-way multilane traffic model for pedestrians in corridors," Networks and Heterogeneous Media, vol. 6, no. 3, pp. 351-381, 2011.

[31] R. L. Hughes, "The flow of human crowds," Annual Review of Fluid Mechanics, vol. 35, no. 1, pp. 169-182, 2003.

[32] Y.-Q. Jiang, P. Zhang, S. C. Wong, and R.-X. Liu, "A higher-order macroscopic model for pedestrian flows," Physica A: Statistical Mechanics and Its Applications, vol. 389, no. 21, pp. 4623-4635, 2010.

[33] B. Piccoli and A. Tosin, "Time-evolving measures and macroscopic modeling of pedestrian flow," Archive for Rational Mechanics and Analysis, vol. 199, no. 3, pp. 707-738, 2011.

[34] N. Bellomo and C. Dogbe, "On the modelling crowd dynamics from scaling to hyperbolic macroscopic models," Mathematical Models and Methods in Applied Sciences, vol. 18, supplement 1, pp. 1317-1345, 2008.

[35] E. Cristiani, B. Piccoli, and A. Tosin, "Multiscale modeling of granular flows with application to crowd dynamics," Multiscale Modeling \& Simulation, vol. 9, no. 1, pp. 155-182, 2011.

[36] A. Chertock, A. Kurganov, A. Polizzi, and I. Timofeyev, "Pedestrian flow models with slowdown interactions," Mathematical Models and Methods in Applied Sciences, vol. 24, no. 2, pp. 249275, 2014.

[37] M. Di Francesco and M. Rosini, "Rigorous derivation of nonlinear scalar conservation laws from follow-the-leader type models via many particle limit," Archive for Rational Mechanics and Analysis, 2015.

[38] G. G. Løvås, "Modeling and simulation of pedestrian traffic flow," Transportation Research Part B: Methodological, vol. 28, no. 6, pp. 429-443, 1994.

[39] S. Hoogendoorn and P. H. Bovy, "Simulation of pedestrian flows by optimal control and differential games," Optimal Control Applications \& Methods, vol. 24, no. 3, pp. 153-172, 2003. 
[40] S. J. Older, "Movement of pedestrians on footways in shopping streets," Traffic Engineering \& Control, vol. 10, pp. 160-163, 1968.

[41] S. Milgram and H. Toch, "Collective behavior: crowds and social movements," in The Handbook of Social Psychology, vol. 4, 1969.

[42] T. Kretz, A. Grünebohm, M. Kaufman, F. Mazur, and M. Schreckenberg, "Experimental study of pedestrian counterflow in a corridor," Journal of Statistical Mechanics: Theory and Experiment, no. 10, Article ID P10001, 2006.

[43] D. Helbing and T. Vicsek, "Optimal self-organization," New Journal of Physics, vol. 1, no. 1, article 13, 1999.

[44] G. F. Lawler, Random Walk and the Heat Equation, vol. 55, American Mathematical Society, 2010. 


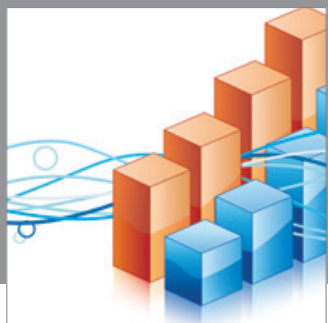

Advances in

Operations Research

mansans

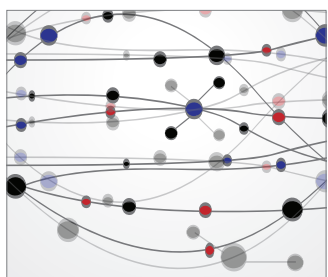

The Scientific World Journal
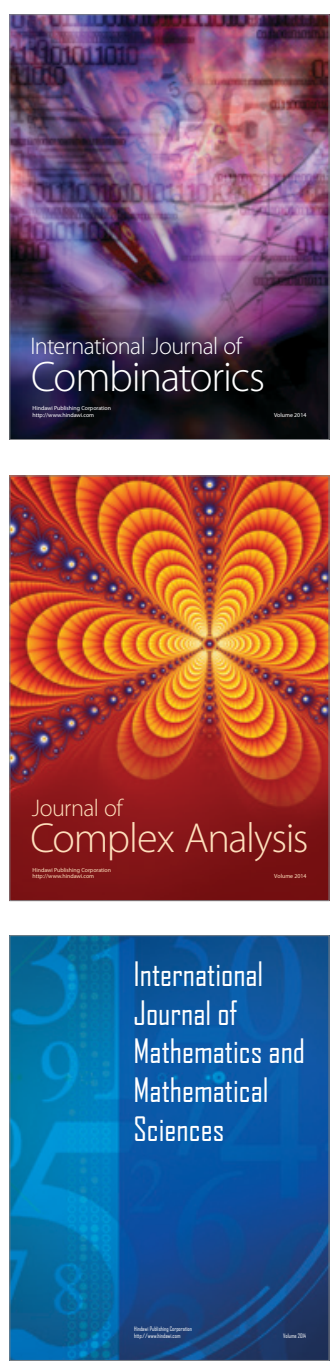
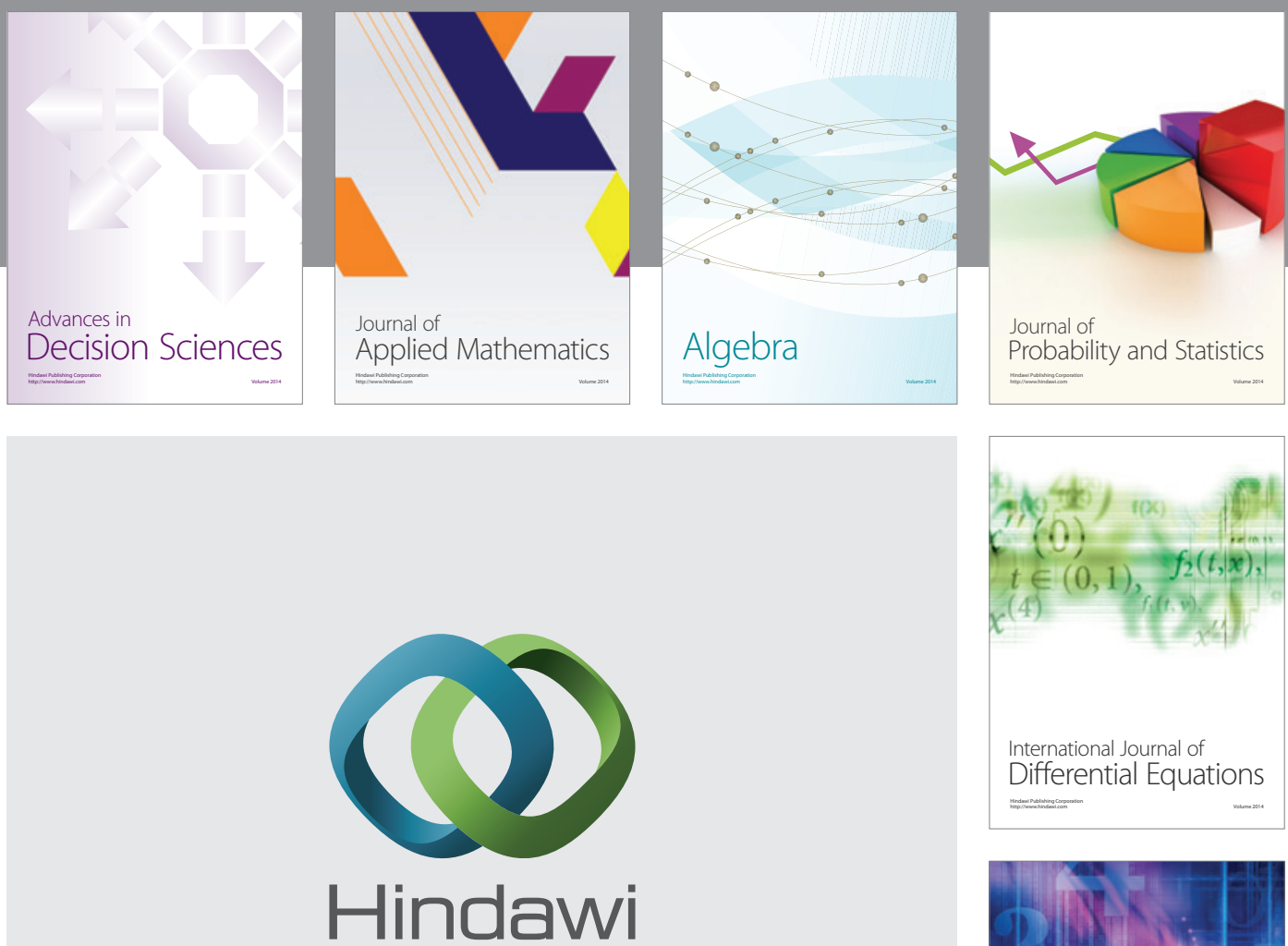

Submit your manuscripts at http://www.hindawi.com
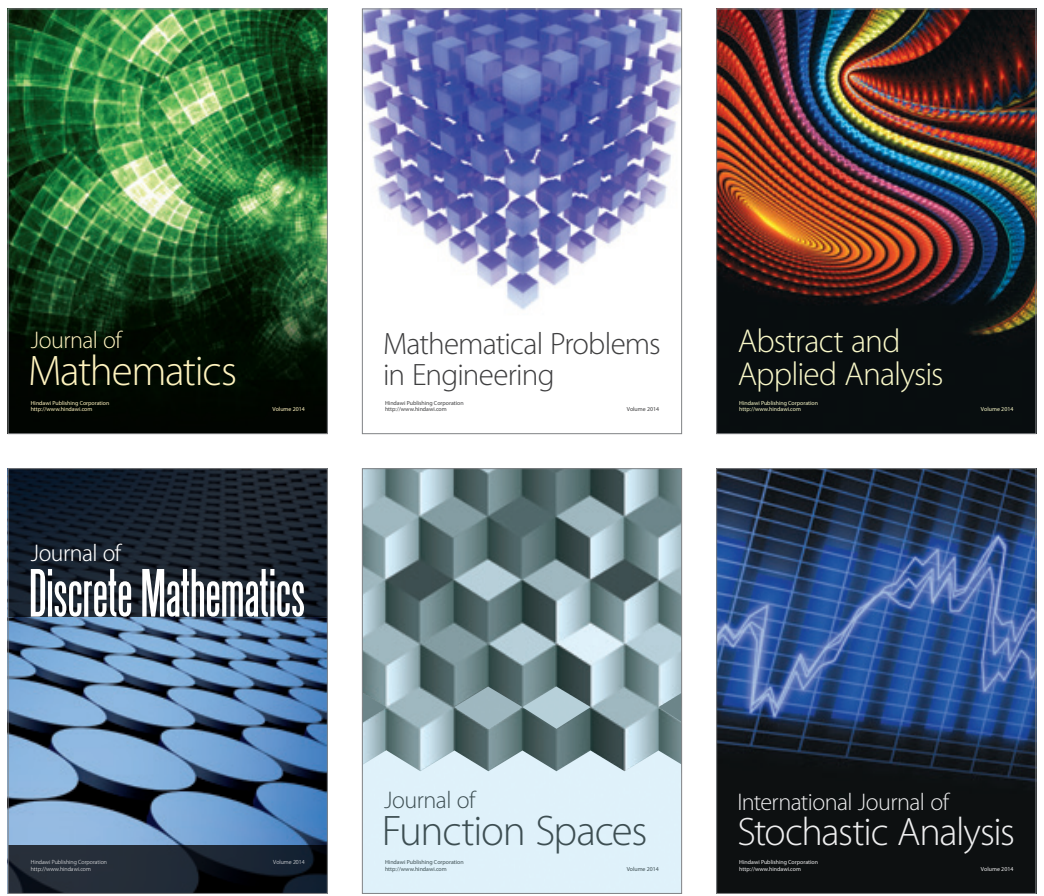

Journal of

Function Spaces

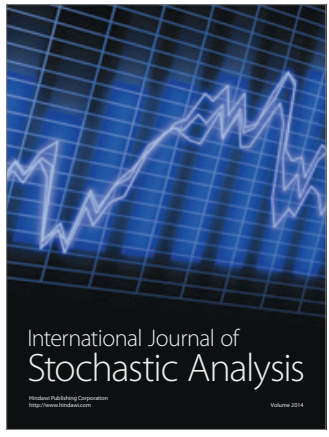

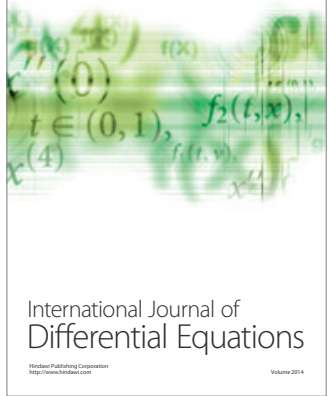
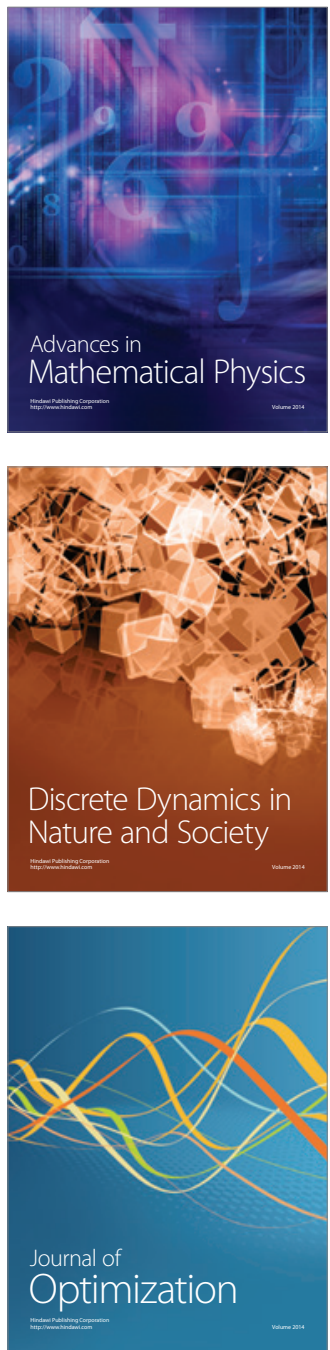